\title{
SPACE: the spectroscopic all-sky cosmic explorer
}

A. Cimatti - M. Robberto - C. Baugh .

S. V. W. Beckwith · R. Content · E. Daddi ·

G. De Lucia • B. Garilli • L. Guzzo • G. Kauffmann •

M. Lehnert • D. Maccagni · A. Martínez-Sansigre •

F. Pasian · I. N. Reid · P. Rosati · R. Salvaterra •

M. Stiavelli • Y. Wang • M. Zapatero Osorio •

M. Balcells • M. Bersanelli • F. Bertoldi • J. Blaizot •

D. Bottini - R. Bower - A. Bulgarelli - A. Burgasser •

C. Burigana - R. C. Butler - S. Casertano - B. Ciardi •

M. Cirasuolo - M. Clampin - S. Cole - A. Comastri •

S. Cristiani - J.-G. Cuby • F. Cuttaia - A. De Rosa •

A. Diaz Sanchez • M. Di Capua - J. Dunlop • X. Fan •

A. Ferrara - F. Finelli - A. Franceschini - M. Franx •

P. Franzetti · C. Frenk • Jonathan P. Gardner •

F. Gianotti - R. Grange • C. Gruppioni - A. Gruppuso •

F. Hammer · L. Hillenbrand - A. Jacobsen • M. Jarvis •

R. Kennicutt • R. Kimble • M. Kriek • J. Kurk •

J.-P. Kneib • O. Le Fevre • D. Macchetto •

J. MacKenty • P. Madau • M. Magliocchetti • D. Maino •

N. Mandolesi - N. Masetti · R. McLure - A. Mennella •

M. Meyer • M. Mignoli • B. Mobasher • E. Molinari •

G. Morgante - S. Morris - L. Nicastro · E. Oliva •

P. Padovani - E. Palazzi - F. Paresce ·

A. Perez Garrido - E. Pian - L. Popa - M. Postman •

L. Pozzetti - J. Rayner • R. Rebolo • A. Renzini •

H. Röttgering • E. Schinnerer • M. Scodeggio •

M. Saisse • T. Shanks • A. Shapley • R. Sharples •

H. Shea · J. Silk · I. Smail • P. Spanó • J. Steinacker •

L. Stringhetti • A. Szalay • L. Tresse - M. Trifoglio •

M. Urry • L. Valenziano • F. Villa • I. Villo Perez •

F. Walter • M. Ward - R. White - S. White - E. Wright •

R. Wyse - G. Zamorani - A. Zacchei - W. W. Zeilinger •

F. Zerbi

Received: 30 November 2007 / Accepted: 9 April 2008 / Published online: 24 May 2008

(C) Springer Science + Business Media B.V. 2008

A. Cimatti $(\bowtie)$

Dipartimento di Astronomia, Università di Bologna, Bologna, Italy

e-mail: a.cimatti@unibo.it

M. Robberto $\cdot$ S. V. W. Beckwith · I. N. Reid · M. Stiavelli .

S. Casertano - J. MacKenty · M. Postman · R. White

Space Telescope Science Institute, Baltimore, MD, USA 
Abstract We describe the scientific motivations, the mission concept and the instrumentation of SPACE, a class-M mission proposed for concept study at the first call of the ESA Cosmic-Vision 2015-2025 planning cycle. SPACE aims to produce the largest three-dimensional evolutionary map of the Universe over the past 10 billion years by taking near-IR spectra and measuring redshifts for more than half a billion galaxies at $0<z<2$ down to $A B \sim 23$ over $3 \pi$ sr of the sky. In addition, SPACE will also target a smaller sky field, performing a deep spectroscopic survey of millions of galaxies to $A B \sim 26$ and at $2<z<10+$. These goals are unreachable with ground-based observations due to the $\approx 500$ times higher sky background (see e.g. Aldering, LBNL report number LBNL-51157, 2001). To achieve the main science objectives, SPACE will use a $1.5 \mathrm{~m}$ diameter RitcheyChretien telescope equipped with a set of arrays of Digital Micro-mirror Devices covering a total field of view of $0.4 \mathrm{deg}^{2}$, and will perform large-multiplexing multi-object spectroscopy (e.g. $\approx 6000$ targets per pointing) at a spectral resolution of $\mathrm{R} \sim 400$ as well as diffraction-limited imaging with continuous coverage from

C. Baugh · R. Bower · S. Cole · C. Frenk · S. Morris · T. Shanks · I. Smail · M. Ward Institute of Computational Cosmology, University of Durham, Durham, UK

R. Content $\cdot$ R. Sharples

Centre for Advanced Instrumentation, University of Durham, Durham, UK

E. Daddi

CEA Saclay, Gif-sur-Yvette Cedex, France

G. De Lucia · G. Kauffmann · J. Blaizot · B. Ciardi · S. White

MPA Garching, Garching, Germany

B. Garilli $\cdot$ D. Maccagni $\cdot$ D. Bottini $\cdot$ P. Franzetti $\cdot$ M. Scodeggio

IASFMI, INAF, Rome, Italy

L. Guzzo · E. Molinari · P. Spanó · F. Zerbi

Brera, INAF, Rome, Italy

M. Lehnert · F. Hammer

Observatoire de Paris - Meudon, Paris, France

A. Martínez-Sansigre · J. Kurk · E. Schinnerer · J. Steinacker · F. Walter

Max-Planck-Institut für Astronomie Heidelberg, Heidelberg, Germany

F. Pasian · S. Cristiani · M. Magliocchetti · E. Pian · A. Zacchei

Osservatorio Astronomico di Trieste, INAF, Rome, Italy

P. Rosati · P. Padovani

ESO Garching, Garching, Germany

R. Salvaterra · M. Bersanelli · D. Maino - A. Mennella

University of Milano, Milan, Italy

Y. Wang

University of Oklahoma, Norman, OK, USA

M. Zapatero Osorio · M. Balcells · R. Rebolo

IAC, Santa Cruz de Tenerife, Spain

亚 Springer 
0.8 to $1.8 \mu \mathrm{m}$. Owing to the depth, redshift range, volume coverage and quality of its spectra, SPACE will reveal with unique sensitivity most of the fundamental cosmological signatures, including the power spectrum of density fluctuations and its turnover. SPACE will also place high accuracy constraints on the dark energy equation of state parameter and its evolution by measuring the baryonic acoustic oscillations imprinted when matter and radiation decoupled, the distanceluminosity relation of cosmological supernovae, the evolution of the cosmic expansion rate, the growth rate of cosmic large-scale structure, and high- $z$ galaxy clusters. The datasets from the SPACE mission will represent a long lasting legacy for the whole astronomical community whose data will be mined for many years to come.

Keywords Observational cosmology • Dark energy • Astronomical and space-research instrumentation

PACS 98.80.Es • 95.36. $+\mathrm{x} \cdot 95.55 .-\mathrm{n}$

F. Bertoldi

University of Bonn, Bonn, Germany

A. Bulgarelli · C. Burigana · R. C. Butler · F. Cuttaia · A. De Rosa · F. Finelli · F. Gianotti A. Gruppuso · N. Mandolesi - N. Masetti · G. Morgante - L. Nicastro · E. Palazzi · F. Paresce · L. Stringhetti · M. Trifoglio · L. Valenziano · F. Villa IASFBO, INAF, Rome, Italy

A. Burgasser MIT, Cambridge, MA, USA

M. Cirasuolo · J. Dunlop · R. McLure ROE, Edinburgh, UK

M. Clampin - J. P. Gardner · R. Kimble GSFC, NASA, Washington, D.C., USA

A. Comastri · C. Gruppioni · M. Mignoli · L. Pozzetti · G. Zamorani Osservatorio Astronomico di Bologna, INAF, Bologna, Italy

J.-G. Cuby · R. Grange · J.-P. Kneib · O. Le Fevre · M. Saisse · L. Tresse LAM, Marseille, France

A. Diaz Sanchez · A. Perez Garrido · I. Villo Perez UPCT, U. Politecnica de Cartagena, Cartagena, Spain

M. Di Capua

UMD, College Park, MD, USA

J. Dunlop

CRC, Ottawa , Canada

X. Fan

University of Arizona, Tucson, AZ, USA 


\section{Introduction and scientific background}

Our view of the Universe has changed dramatically over the past two decades through measurements of the cosmic microwave background (CMB), the large-scale structure of the local Universe $(z<0.3)$ and the brightness of distant supernovae. Fully $96 \%$ of the constituents consist of non-luminous and unidentified dark energy (73\%) and dark matter (23\%) that govern the expansion history and evolution of cosmic structure and leave their imprints on the structure and distribution of visible galaxies. These dark components are unexplained in standard physical theory, but are, nevertheless, considered a natural feature of standard cosmology because they can explain a wide range of observations assuming only that dark energy produces a pressure countering gravity and dark matter behaves like ordinary matter in its effect on space-time.

The acceleration of the expansion of the Universe is considered one of the most important discoveries of cosmology [40, 41]. Both of the extant explanations for accelerating space-time require new physics: a negative pressure component dubbed dark energy, or a modification of the law of gravity and, therefore, the standard framework underpinning cosmology. The first possibility, dark energy,

A. Ferrara

SISSA, Trieste, Italy

A. Franceschini

University of Padova, Padua, Italy

M. Franx · M. Kriek · H. Röttgering

University of Leiden, Leiden, The Netherlands

L. Hillenbrand

Caltech, Pasadena, CA, USA

A. Jacobsen

OpSys Project Consulting, Schoeffengrund, Germany

M. Jarvis

University of Hertfordshire, Hatfield, UK

R. Kennicutt

IoA, Cambridge, UK

D. Macchetto

ESA, Paris, France

P. Madau

UCSC, Santa Cruz, CA, USA

M. Meyer

Steward Observatory, Tucson, AZ, USA

B. Mobasher

UC Riverside, Riverside, CA, USA

E. Oliva

Osservatorio Astrofisico di Arcetri, INAF, Florence, Italy

首 Springer 
is currently favoured but, nevertheless, provides little help because we have no plausible candidates from elementary particle theories [37]. The simplest case is represented by the so called cosmological constant, $\Lambda$. Unfortunately, there is no theoretical justification for the size of the cosmological constant as inferred from the rate of universal acceleration. Arguments based on the standard model of particle physics yield values between $10^{50}$ and $10^{123}$ times larger than the observed value. The cosmological constant is only one of several candidates to explain the acceleration of the universe. Most possibilities can be parameterized by their equation of state, the ratio of the pressure exerted by the dark energy to the energy density of the field: $w=P / \rho c^{2}$. A cosmological constant necessarily implies $w=-1$. The simplest alternative quantum-field explanations allow $w$ to differ from -1 and to vary with time. Translating from linear time to observable redshift, $z$, we can parameterize $w(z)=w_{0}+w_{a}(1-a), a=1 /(1+z)$. The magnitude of $w$ determines the rate of acceleration (through the associated pressure), so it can be characterized by measuring the rate of acceleration as a function of time for the history of the Universe, or equivalently, the Hubble parameter as a function of redshift, $H(z)$. Alternatively, the acceleration could be the result of a modified form of gravity, such as gravity that is a function of scale (e.g. [13]), or a modification of the standard model through string or brane theories (e.g. [38]). Distinguishing between dark energy, modified gravity and different variants necessarily requires high-precision measurements of the cosmic

E. Oliva

TNG, Rome, Italy

L. Popa

University of Bucharest, Bucharest, Romania

J. Rayner

Institute for Astronomy, Honolulu, HI, USA

A. Renzini

Osservatorio Astronomico di Padova, INAF, Padova, Italy

A. Shapley

Princeton, Princeton, NJ, USA

H. Shea

Ecole Polytechnique Federale de Lausanne, Lausanne, Switzerland

J. Silk

University of Oxford, Oxford, UK

A. Szalay $\cdot$ R. Wyse

Johns Hopkins University, Baltimore, MD, USA

M. Urry

Yale, New Haven, CT, USA

E. Wright

UCLA, Los Angeles, CA, USA

W. W. Zeilinger

University of Vienna, Vienna, Austria 
expansion rate history $H(z)$, but the remaining differences among theories mean that even a very accurate $H(z)$ is inadequate to uniquely isolate one theory (e.g. $[28,55,58])$. The degeneracy occurs because a dark energy model and a modified gravity theory can give identical cosmic expansion histories $\mathrm{H}(\mathrm{z})$, but would give very different growth histories of cosmic large scale structure [30]. However, the predicted growth rate of large-scale structures varies among theories, and a measurement of structure evolution can isolate the correct one. The best current constraints on $w$ from observations of distant supernovae are consistent with a cosmological constant: $-1<w<-0.85$ with $10 \%-20 \%$ uncertainties assuming a flat Universe [42, 45, 67].

The cosmological models make testable predictions about how the dark components affect a wide range of observable features that can be discriminated with high precision only using newly developed technology on spacecraft. In this framework, we designed a new space mission aimed at addressing the key questions of modern cosmology. This study led to a proposal that was submitted to ESA in response to the first call Cosmic Vision 2015-2025: the SPectroscopic All-sky Cosmic Explorer $\left(S P A C E^{1}\right)$. To solve the mystery of dark energy, SPACE will determine $H(z)$ and constrain $w_{0}$ and $w_{a}$ by combining the observations of the Baryonic Acoustic Oscillations (BAO), the growth rate of structures, distant Type Ia Supernovae and high redshift galaxy clusters. But SPACE will also address several other key questions of modern cosmology besides dark energy. The main scientific objectives of SPACE are presented in the next sections.

\section{$2 S P A C E$ and the power spectrum of density fluctuations}

Thanks to the enormous volume sampled and the availability of spectroscopic redshifts, SPACE will be able to place constraints at an unprecedented level on the power spectrum of matter fluctuations, $P(k)$, which is a key input to theoretical models of structure formation. The spectrum of CMB temperature fluctuations, $C_{l}$, and the matter $P(k)$ are subject to different parameter degeneracies. While CMB spectra depend on the combination $\Omega_{m} h^{2}$, the matter $P(k)$ is sensitive to $\Omega_{m} h$. Thus, combining these two datasets help to break degeneracies and adds tremendous scope to tighten the limits on parameters which result from the use of just one dataset in isolation. The $C_{l}$ spectrum expected from the Planck ${ }^{2}$ satellite will probe to smaller angular scales than WMAP, ${ }^{3}$ significantly increasing the overlap with the scales probed by the SPACE measurement of $P(k)$. The massive volume covered by SPACE means that we will be able to make the first compelling measurement of the turnover in the matter $P(k)$. This feature is a prediction of the standard paradigm of structure formation, imprinted when matter first started to dictate the expansion of the Universe around 27,000 years after the Big Bang. This measurement of the large scale power will be the first direct probe of primordial fluctuations, which have only been measured to date through ripples in the CMB. The position and shape of the turnover in $P(k)$ depends upon the matter density of the universe, and how much of

\footnotetext{
${ }^{1}$ http://urania.bo.astro.it/cimatti/space.

${ }^{2}$ http://www.rssd.esa.int/Planck.

${ }^{3} \mathrm{http} / / / \mathrm{map} . g s f c . n a s a . g o v /$.

Springer
} 
this mass is in the form of baryons and massive neutrinos. By measuring the slope of the power spectrum on scales larger than the turnover we will be able to distinguish between different models of inflation, extending the grasp of SPACE on the history of the universe right back to within a tiny fraction second after the Big Bang.

\section{SPACE and BAOs}

BAO are small amplitude (5-10\%) modulations in the distribution of matter imprinted at the epoch when matter and radiation decoupled $(z \sim 1000)$. These ripples correspond to sound waves in the primordial photon-baryon fluid. The length scale of the oscillations is closely related to the sound horizon at decoupling, a scale $(\sim 150 \mathrm{Mpc})$ which is accurately known from measurements of CMB temperature fluctuations [53]. Acoustic patterns imprinted on the CMB have provided the most precise measurements of cosmological parameters by WMAP and form the basis for the even more precise measurements anticipated for the Planck. The physical scale of these oscillations will have different apparent sizes on the sky at different redshifts and in different cosmologies. Assuming that the distribution of galaxies reflects the distribution of all matter, this physical scale is measured from the spatial correlation (i.e. the power spectrum) of galaxies. The acoustic oscillation physical scale length is effectively a standard ruler whose apparent size can be measured in the transverse and the radial directions (3D) from the distribution of galaxies. Combining the apparent size with the known physical size gives the distance at the redshift of observation and makes it possible to determine the expansion rate as a function of redshift, $H(z)[8,20,49,62]$. This technique is considered a very promising new method proposed to measure the evolution of the expansion rate and therefore the relative effects of dark energy and dark matter. The application of BAOs to extant galaxy surveys (in combination with other datasets or with restrictive assumptions like a flat Universe) has already yielded constraints on $w$ with uncertainties of only $10 \%$ (e.g. $[15,22,23,39,56])$ even though this technique is only a few years old. The astrophysical effects which may cause deviations of the ideal BAO signature, for example nonlinear evolution of the density field, dynamical redshift-space distortions, galaxy biasing are now well understood from numerical calculations [4, 5, 16, 21, 49, 50]. Angulo and collaborators [5] demonstrated that the primary limitation on the accuracy of the BAO method is sample variance. Thus, to minimize the uncertainty in $w$, the observations need simply to maximize the survey volume. To maximize the sample volume, SPACE will perform a spectroscopic AllSky Survey to measure galaxies over $3 / 4$ of the entire sky and over the redshift interval $0<z<2$, centered on the redshift $z \sim 1$ at which the dark energy first starts to dominate the expansion of the Universe. The sensitivity requirement is $S N R \sim 3$ per resolution element at $H_{\mathrm{AB}} \sim 23$. Taking spectra at near infrared wavelengths ensures that SPACE covers the entire redshift range efficiently. The use of a space-based multi-object spectrometer makes it possible to get accurate redshifts of millions of galaxies over the whole sky in a short time. Figures 1 and 2 show the power of the SPACE BAO approach and the expected accuracy. It is clear that SPACE will dramatically improve our knowledge of $w_{0}$ and $w_{a}$. Recent works emphasized the need to trace the evolution of dark energy, $w(z)$, with high accuracy in order to derive the most stringent constraints on the dark energy (see [28]). 


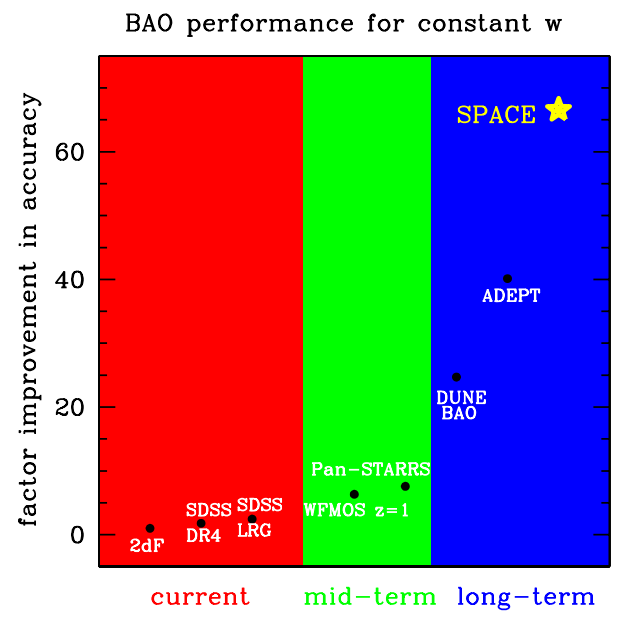

Fig. 1 The relative accuracy with which various BAO experiments are forecast to measure the cosmological constant (or a fixed equation of state for the dark energy, i.e. $\Delta w=\Delta w_{0}$ and $w_{a}=0$ ), without combining with any other cosmological constraints. SPACE will yield a BAO measurement that is over 65 times more accurate than can be made currently with the $2 \mathrm{dF}$ survey, and outperforms any other proposed BAO experiment. The relative accuracy is computed by taking the square root of the ratio of the effective survey volume to the volume of the $2 \mathrm{dF}$

Thanks to the huge volume and wide redshift range covered, SPACE will achieve $0.5 \%$ accuracy in the BAO scale measurement redshift slices with $\Delta z \sim 0.5$. Even more accurate constraints on dark energy will result from a combination of independent and complementary datasets (e.g. see [25]). But even in the case of Planck + SPACE only (see Fig. 2), the constraints on $w$ will be $\approx 5$ (SPACE BAOs), $\approx 10$ (SPACE BAOs + current SNe Ia), and $\geq 20$ (SPACE BAOs + SPACE SNe) times more accurate than the current accuracy. This also highlights the "self-sufficiency" of SPACE.

In 2006, NASA established the Dark Energy Task Force $\left(\mathrm{DETF}^{4}\right.$ with the purpose of studying and defining the accuracies reachable with future experiments dedicated to dark energy.

None of the currently planned ground-based BAO experiments can compete with the accuracy on $w$ reachable with a SPACE-like survey. For instance, the SDSS-III BOSS (Baryon Oscillation Spectroscopic Survey) experiment based on measuring BAOs from Luminous Red Galaxies at $z<0.7$ over a $10,000 \mathrm{deg}^{2}$ sky area (see http://www.sdss3.org/cosmology.php) would achieve a DETF Figure of Merit (FoM) about an order of magnitude less than a SPACE-like survey to $H_{\mathrm{AB}}=23$ over 20,000 $\mathrm{deg}^{2}$ and SPACE fully meets the requirements of the DETF which recommended that the goal for new survey designs should be that their Figure of Merit improves by a factor of three between different Stages of dark energy missions [1]. Combining the SPACE BAO measurement with a long-term Stage IV experiments, such as LSST, JDEM and SKA, produces a further factor of three gain in the constraints on dark energy. Thus, SPACE falls in the next category of Stage $V$ experiments capable

${ }^{4}$ http://home.fnal.gov/ rocky/DETF/. 
Fig. 2 The accuracy on dark energy parameters reachable by combining Planck and SPACE. The ellipses indicate the joint $68 \%$ confidence interval constraints on the dark energy equation of state parameter $\left(w_{0}\right)$ and its evolution with redshift $\left(w_{a}\right)$. The dotted lines refer to the combination of 3-year WMAP data [53], 182 SNe Ia ([42], including [6] data), and SDSS BAO measurements [15] (see [64] for more details)

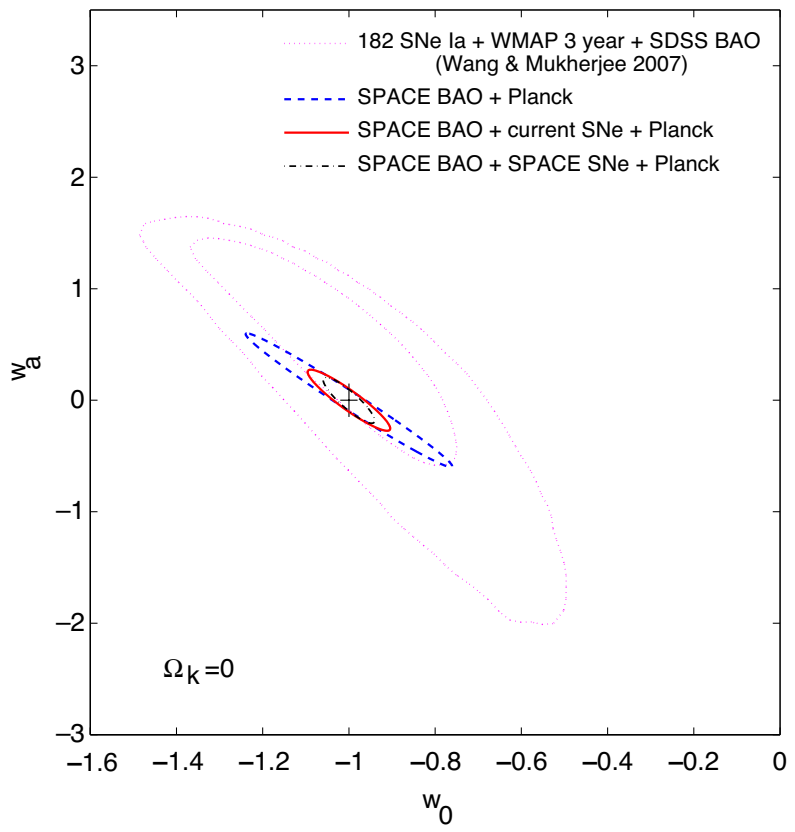

to improve our understanding of the dominant systematic effects in dark energy measurements and, wherever possible, reduce them.

\section{$4 S P A C E$ and the growth rate of cosmic structures}

The growth rate of cosmic structures due to gravitational instability offers the capability to distinguish between different theories that produce the same $H(z)$ and provides an independent and novel probe of the nature of dark energy or possible modifications to gravity. The growth rate of density fluctuations at a given redshift is $f_{g}=d \ln (D) / d \ln (a)$, where $D(a)$ (or $D(t)$ ) is the growing solution of the equation describing the time evolution of density fluctuations (which depends on the gravity theory and on the expansion history). It has been shown that to a good approximation $f$ is well described as $f_{g}(z) \sim \Omega_{m}(z)^{\gamma}$ [27] for a wide range of models, including modifications of General Relativity. $f_{g}(z)$ can be measured from the impact of gravitationally induced peculiar motions (i.e. motions of galaxies in their local rest frame relative to the systemic flow due to the Hubble expansion) on the pattern of galaxy clustering. These motions produce an anisotropy in the galaxy redshiftspace correlation function, $\xi\left(r_{p}, \pi\right)$, in directions parallel and perpendicular to the line of sight. The coherent bulk flows towards overdensities lead to an enhancement of the clustering signal on large scales, quantified as a compression in the isocorrelation levels of $\xi\left(r_{p}, \pi\right)$. The compression is directly proportional to the value of $f_{g}(z)$, modulo the bias factor of the objects used to trace the matter distribution, $b$, measured by the parameter $\beta=f_{g}(z) / b$ (e.g. [19]). The feasibility of this technique at intermediate redshifts has just been demonstrated in an important breakthrough using the VVDS redshift survey and by measuring $\beta$ with $40 \%$ uncertainty from an area of $4 \mathrm{deg}^{2}$ using 6000 galaxy redshifts over a wide redshift range [18] (Pierleoni et al., submitted for publication). Other recent studies emphasized the power of 


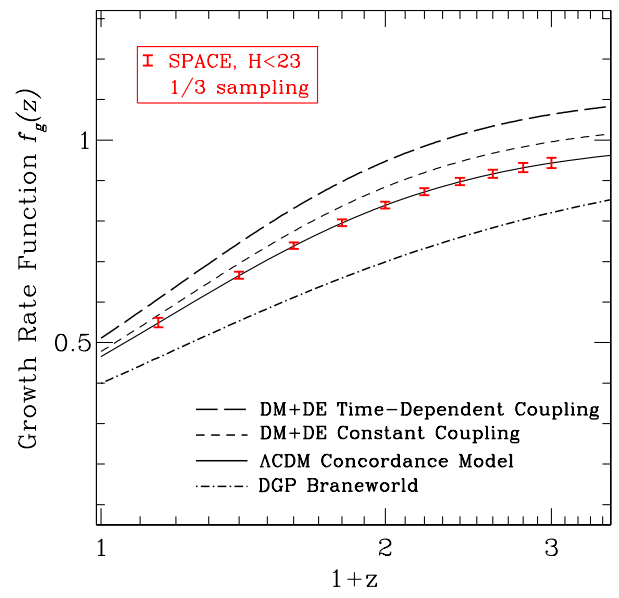

Fig. 3 The predicted performance of SPACE in measuring the growth rate of density fluctuations as a function of redshift, $f_{g}(z)$ using the anisotropy of galaxy correlations, assuming a $\Lambda \mathrm{CDM}$ fiducial model (see [18]). SPACE will disentangle to high accuracy the cosmological constant theory from variants of dark energy (here two possible cases are shown - [3]). The combination of the fully independent measurements of $H(z)$ from BAOs and $f_{g}(z)$ from redshift distortions will provide a direct test of whether cosmic acceleration is due to a modification of Einstein theory of gravitation or to new physics beyond the standard model

this approach for differentiating between dark energy and modified gravity [29, 63]. SPACE will make a dramatic improvement over this, thanks to the enormous volume sampled, even in slices of $\Delta z=0.2$, and to the deep infrared selection $\left(H_{\mathrm{AB}}<23\right)$, that allows the $N(z)$ to extend significantly above $z=1.5$, with a mean density always larger than $\sim 0.02 \mathrm{gal} \mathrm{h}^{3} \mathrm{Mpc}^{-3}$ to $z \sim 2$ even with a sampling of $1 / 3$ galaxies. Our simulations clearly show that SPACE will be unique in providing a precision measurement of the growth history of cosmic structures, reaching $0.5 \%$ accuracy in $\beta$ in redshift slices of $\Delta z=0.2$, and measuring directly the growth function $f_{g}(z)$ to $1 \%$ accuracy (Fig. 3). The bias factor $(b)$ will be extracted in each redshift slice using higher-order galaxy clustering [60] or via self-consistent densityreconstruction methods (e.g. [51]).

\section{SPACE and distant Type Ia Supernovae}

Using Type Ia Supernovae (SNe Ia) as standard candles to measure $H(z)$ is the method by which the accelerating expansion of the Universe was first discovered $[40,41]$. SPACE observational strategy can be designed to allow the discovery of SNe Ia and to obtain their spectroscopic redshifts out to $z \sim 2$ very efficiently thanks to the simultaneous multi-object spectroscopy over a wide field. For instance, the repeated observation of a $4 \mathrm{deg}^{2}$ Deep Field $\left(H_{\mathrm{AB}}<26\right)$ would allow the identification of $\sim 2300$ SNe to $z \sim 2$ in about 5-7 months spread over 1 year, where 1 visit would take 4 days to cover $4 \mathrm{deg}^{2}$ to $H_{\mathrm{AB}} \sim 26$, and each visit would be repeated every 7-10 days. We also note that near-IR spectroscopy is advantageous because less affected by dust extinction, and hence very useful for high-z SNe. The details of the SPACE -SNe program will depend on the developments of other future projects in space and on the ground (e.g. SNAP, Pan-STARRS, LSST, ...). One could imagine 
a scenario where SNAP and SPACE will be operational in the same time frame in order to provide the strong synergy with SNAP discovering the SNe through imaging and SPACE obtaining their spectra and redshifts.

\section{SPACE and high-redshift galaxy clusters}

Galaxy clusters are the end products of the hierarchical build-up of small systems into increasingly larger structures up to $10^{15} \mathrm{M}_{\odot}$. Since clusters arise from rare high peaks of primordial density perturbations, their number densities and mass distribution, i.e. their mass function $n(M, z)$, is highly sensitive to the matter density parameter $\Omega_{m}$, and dark energy parameters $(\Lambda, w)$ which control the rate at which structure grows, as well as to the normalization of the matter power spectrum, $\sigma_{8}$. At $z>1$, the existing surveys do not yet place strong constraints on dark energy due to the small volumes probed and because only a handful of systems have been identified to date (e.g. [36, 43, 54]). SPACE, with its unmatched spectroscopic speed in nearIR, will provide spectroscopic confirmation of all the thousands of clusters at $z>1$ detected in the next generation near-IR, Sunyaev-Zeldovich (SZ) and X-ray large area surveys (e.g. SPT in 2007+, Planck in 2008+, eROSITA in 2012+). SPACE will identify bona-fide virialized structures, the ones to be compared to the theoretical cluster mass function, and it will resolve the cases of contamination from point-like radio sources and AGN that are expected to plague future SZ and X-ray searches for distant clusters. Thus, SPACE will unleash the full potential of the next generation cluster surveys by allowing the best possible knowledge of systematic errors, with small statistical uncertainties limited only by the volume of the observable Universe. We recall that constraints from cluster surveys generally exhibit parameter degeneracies different from those of other techniques, such as CMB, Type-Ia SNe and BAO described above. The pursuit of these complementary means, based on different physical properties of the Universe, is the most powerful way to unveil the nature of the dark energy and dark matter. In addition, SPACE itself, thanks to the unbiased near-infrared selection and the spectroscopic survey will locate for the first time several tens of thousands of clusters directly in three dimensions out to $z \sim 2.5$ and over a large mass range.

\section{From dark matter to baryons: galaxy formation and evolution}

In the standard model of galaxy formation, galaxies coalesce from gas that cools inside dark matter halos $[7,65]$. Determining the physical processes that regulate the growth of galaxies and their link with massive black holes is one of the outstanding problems of modern astrophysics. By observing galaxies down to faint magnitudes and sampling randomly one of every three of these faint galaxies, SPACE will measure the characteristics of more than half a billion galaxies as a function of their environment at sensitivities impossible to obtain from ground-based telescopes. We take this opportunity to underline why SPACE must observe in the near-infrared. The strong optical-ultraviolet spectral features of galaxies well below $L_{*}$ that are primary redshift and diagnostic tracers fall, in the redshift range $z \sim 0.5-3$, in the near-IR. Their list includes: Balmer and D4000 continuum breaks, strong H Balmer 
lines, CaII H\&K lines, as well as the well-studied diagnostic emission lines such as [O II] $\lambda 3727$, Balmer lines, [O III] $\lambda \lambda 4955,5007$, and several high ionization lines useful for identifying the presence of an active galactic nucleus. At higher redshifts, SPACE will obtain redshifts and diagnostics of the young stellar populations and the interstellar medium using rest-frame ultraviolet lines. SPACE 's combination of wavelength coverage, resolution and sensitivity will remove the degeneracy limiting the photometric SED fitting (reddening vs. red stars). Examples of what SPACE will be able to accomplish include: (1) the evolution of the distribution functions with the highest accuracy possible and limited only by cosmic variance (e.g. luminosity, stellar mass) significantly below the characteristic mass, $M_{*}$, (2) the cosmic evolution of $\mathrm{red} /$ passive galaxies at $0<z<2$ and up to their formation epoch, (3) to investigate how the properties of galaxies depend on the density of their surroundings, (4) to determine the merger rate as a function of redshift, (5) to determine black hole masses from line widths and compare them to the stellar content, mass, and age of the host galaxy, to directly probe feedback processes in the largest ever high-z sample of AGN, (6) to determine the causes of "downsizing", whereby starformation histories correlate with stellar mass, and the active sites of star-formation move to increasingly higher mass galaxies with redshift, (7) to provide a unique resource for measuring all aspects of the spatial distribution of galaxies and clusters on smaller scales, including the higher order correlation functions (i.e. the amplitudes of the $\mathrm{N}$-point correlation functions) which are sensitive to the growth of structure through gravitational instability and offer a direct route to measuring galaxy bias (see Section 4). Moreover, SPACE will enable new facilities such as LOFAR, eROSITA, WISE, SKA to reach their full capability by removing the significant bottleneck in obtaining the redshifts for the large survey samples.

\section{Galaxies and QSOs in the early universe}

In the area of galaxy and AGN co-evolution, SPACE will provide a unique contribution through a spectroscopic Deep Survey, by targeting galaxies at redshifts as high as 10, when the Universe was a few hundred million years old. The SPACE Deep Survey will be designed to study large samples at high redshifts following a strategy of this type: (1) $Z$-, $J$ - and $H$-band imaging of a $10 \mathrm{deg}^{2}$ area to $H=26, J \sim 28, Z \sim 28$, (2) narrow-band imaging of the same area, (3) spectroscopy to $H=25$ over the same area and with a sampling rate of $90 \%$ (mostly at $2<z<7$ ), (4) the candidates at $z>7$ will be pre-selected from broad-band colors [10-12] using the SPACE imaging data themselves and will be repeatedly observed for secure spectroscopic identification. Based on the observed galaxy luminosity function at $z=6$ [10], extrapolated at higher redshift under the assumption of simple luminosity evolution, in an area of $10 \mathrm{deg}^{2}$, there will be $\sim 800$ galaxies at $z>7$ with $H<26^{m}, \sim 500$ at $7<z<8$, $\sim 200$ at $8<z<9$, and a few tens above $z=9$. As the most massive objects at these redshifts are extremely rare, the large FOV of SPACE compared to JWST gives it an enormous advantage: only $\sim 0.3$ massive galaxies should be present in the JWST field of view of $\sim 10 \mathrm{arcmin}^{2}$. SPACE is the natural complement to JWST for the study of high redshift galaxies. With narrow-band filters, it will be possible to search for Ly- $\alpha$ emitters too faint to be detected in the broad-band SPACE images by selecting objects showing an excess of flux in narrow-band filters with respect to 
the broad-band one. For instance, filters centered at $\sim 1.03$ and $\sim 1.34 \mu \mathrm{m}$, allowing an extensive Ly- $\alpha$ emitter search at $z \sim 7.7$ and $z \sim 10$. At $A B=26$, SPACE should find $\sim 1200(\sim 30)$ emitters at $z \sim 7.7(z \sim 10)$ objects with Ly- $\alpha$ fluxes as low as $\sim 4 \times 10^{-18} \mathrm{erg} \mathrm{s}^{-1} \mathrm{~cm}^{-2}$ with $S / N \sim 10$. A fraction (up to $10 \%$ ) of these sources is expected to be powered by very massive, metal-free stars, the so called Population III stars [46]. These objects can be selected from SPACE data owing to their very large Ly- $\alpha$ equivalent width and He II $\lambda 1640$ emission [47]. Finally, SPACE will also obtain the definitive measurement and spectrum of the so far elusive cosmic Near Infrared Background (NIRB) (e.g. [24] and [57] and references therein). Sources at $z>5$ contributing to the reionization of the Universe are expected to produce a strong feature in the NIRB, with a well defined maximum at $\sim 1 \mu \mathrm{m}$ with $v I_{v} \sim 1 \mathrm{nW} \mathrm{m}^{2} \mathrm{sr}^{-1}$ and a strong break below $1 \mu \mathrm{m}$ due to the strong absorption by the intergalactic medium (IGM) below rest-frame $912 \AA$ [14, 44].

The enormous sky coverage of the SPACE all-sky survey will also reveal several hundred QSOs at $z>6$ (including a few tens of objects at $z>9$ ) and shed light on the evolution of supermassive black holes. To estimate the detected number of high-redshift QSOs, a $z=6$ luminosity function was constructed using current observational constraints $[17,66]$, assuming $2 / 3$ of the sky is covered, and $1 / 3$ sampling rate within this sky coverage. Three scenarios were considered, in order to model the evolution of the $z \geq 6$ QSO LF: no evolution from the $z=6 \mathrm{LF}$, linear evolution, and Eddington (exponentially decaying) evolution with an $e$-folding time of $\tau=45$ Myr. These assumptions yield a detection rate of approximately 200, 150 and $\leq 1(70,30,<1)$ QSOs at $z=7(z>9)$. The SPACE spectral resolution is good enough to study the absorption of photons from $\operatorname{Ly} \alpha$ and $\operatorname{Ly} \beta$ transitions through the IGM as a function of redshift. The masses of QSOs can be measured from the SPACE spectra using the C IV $\lambda 1549 \AA$ Aine [61]. Currently, it takes about $8 \mathrm{~h}$ with a $8 \mathrm{~m}$ telescope to obtain a suitable near-infrared spectrum of a QSO at $z \sim 6$ (e.g. [52]), whereas in 15 min SPACE would go 3 magnitudes fainter. Before 2017, programs on ground-based telescopes will search for high-redshift QSOs by imaging in several optical and near-infrared bands, and using drop-out techniques. However, such techniques are limited by (a) the near-infrared imaging depth achievable from the ground for a large sky area, (b) the limited redshift ranges available to the drop-out technique, (c) the large fraction $(>95 \%)$ of drop out candidates that are cool stars, and (d) the $\mathrm{OH}$ sky lines covering $>40 \%$ of the near-infrared spectrum. Ground-based surveys are expected to find only a relatively small number of highredshift QSOs (for a recent example of the process, see [59]). For a significant increase in the number of objects, and to really open up the unexplored redshift range $(z>7)$, we need to search for high-redshift QSOs over the entire available all sky with spectra that cover a large wavelength range free from by atmospheric emission lines.

\section{The near-infrared view of our milky way galaxy}

At low galactic latitudes source crowding represents a problem for multi-slit spectroscopic surveys. However, the extreme versatility of the instrument we have envisioned allows us to perform integral field spectroscopy using coded masks (Hadamard transforms). A SPACE Galactic-plane survey will be feasible with a 
relatively modest investment of time by covering in integral field mode the strip within $\pm 0.5^{\circ}$ centered on the Galactic Equator between $\pm 60^{\circ}$ of galactic longitude to magnitude $A B \sim 20$. Other examples of key contributions of SPACE in Galactic studies include the census of ultracool dwarfs in the vicinity of the Sun and the estimate of the lower mass function in star-forming regions.

\section{$10 S P A C E$ vs ground-based observations}

All the scientific goals described above need the coverage of the largest possible volume of the Universe, and are reachable within reasonable timescales only if the observations are done from space and in the near-IR by taking advantage of the sky background lower by a factor of $\approx 500$ than from the ground, and the lack of $\mathrm{OH}$ emission lines and telluric absorptions. From the ground, 30\% of the wavelength range between 1 and $2 \mu \mathrm{m}$ is invisible, and for the remaining fraction the bright and variable night sky lines dominate (at the $\sim 95 \%$ level !) the background. At low spectral resolution $(R \sim 500)$, their filling factor is virtually $100 \%$. Even at $R \sim 2000$, less than half the near-IR spectral range is free from night sky line emission [34]. These emission lines, variable in intensity on time scales of minutes, add background noise, subtraction residuals and scattering that make spectral line and redshift determination of faint sources from the ground nearly impossible, no matter how long one integrates. The SPACE uninterrupted spectral coverage of $\sim 0.8-1.8 \mu \mathrm{m}$ coupled with the near-IR selection of the all-sky survey sample $\left(H_{\mathrm{AB}}<23\right)$ ensures a survey speed, a redshift coverage of $0<z<2$ and a spectroscopic redshift measurement success rate that would be impossible to obtain from the ground. This huge advantage opens also the possibility to perform a SPACE deep and wide-field $\left(\approx 10 \mathrm{deg}^{2}\right)$ spectroscopy survey down to limiting magnitudes $\left(H_{\mathrm{AB}}<25-26\right)$ (see Section 4$)$ that would be completely unreachable with groundbased observations even in the era of extremely large telescopes ( $>10 \mathrm{~m}$ diameter).

\section{Simulations}

In order to assess the mission feasibility and efficiency, and to define the most suitable characteristics of the instrumentation, the performances of SPACE were extensively simulated both for imaging and spectroscopy.

\subsection{Spectroscopy}

In the case of spectroscopy, we explored different instrument configurations using the VVDS slit positioning software [9] and using both real and artificial (from the Millennium Simulations) galaxy catalogs with magnitudes matched to the sensitivity limits of the All-Sky and Deep Survey. The software does not allow the overlap of spectra both in the spatial and in the wavelength directions (Fig. 4). The mission efficiency was evaluated as a function of instrumental parameters and galaxy properties, conservatively allowing 2 empty pixels between spectra to accommodate spectral distortions. The best results are obtained with: $4 \mathrm{k} \times 4 \mathrm{k}$ detector, pixel size $0.375^{\prime \prime}$, $15 \AA$ Å/pix dispersion, 2 pixels per Digital Micro-mirror Device (DMD) along disper- 


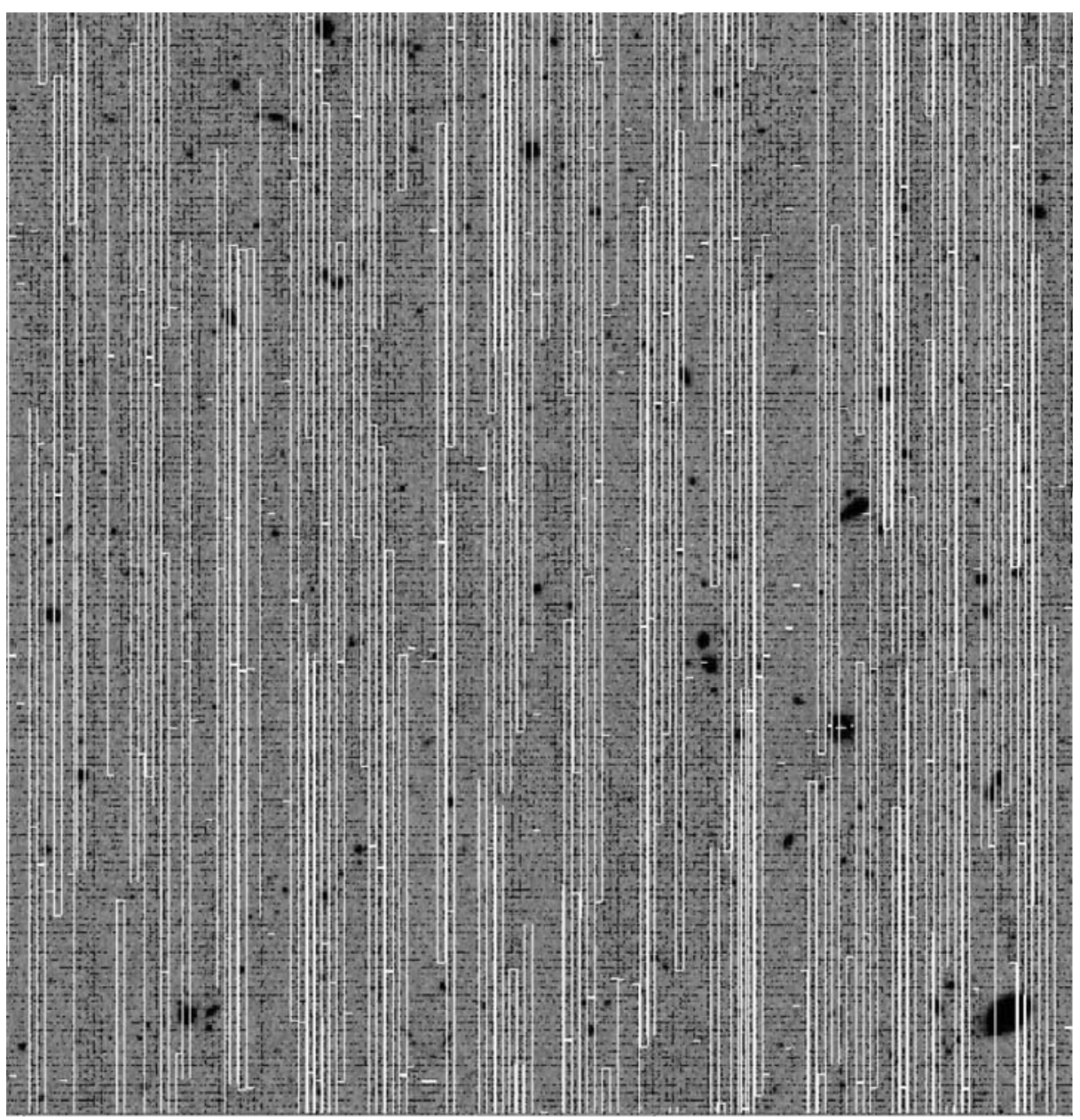

Fig. 4 A simulated $5 \times 7$ field showing the location of the spectra of the selected targets

sion, spectrum length of 670 pixel, spectral resolution of 400. This combination allows a sampling of about 1 of every 3 galaxies to $H_{\mathrm{AB}}=23(\mathrm{AB})$, giving a $\approx 33 \%$ efficiency.

With the above parameters, we simulated spectra including background and detector noise. Template spectra [26, 33, 48] of a variety of galaxy types (earlytype, star-forming, starbursts, AGN, Lyman-break, etc.) provided the input to the simulations as a function of the magnitude and redshift for a total of about 180,000 spectra at $0.2<z<2.4$ for the all-sky survey $\left(H_{\mathrm{AB}}<23\right)$ and about 1,000 at $2.4<z<10$ for the Deep Survey $\left(H_{\mathrm{AB}}<25-26\right)$. The results show that the dispersion and the resolution of SPACE are adequate to reliably identify the main emission and absorption features of all galaxy types (Fig. 5). Redshifts have been automatically measured with EZ (the software tool used in VVDS and zCosmos projects) and other tools specifically developed for the simulations. The low background, wide wavelength coverage, and moderate spectral resolution produce a success rate of 


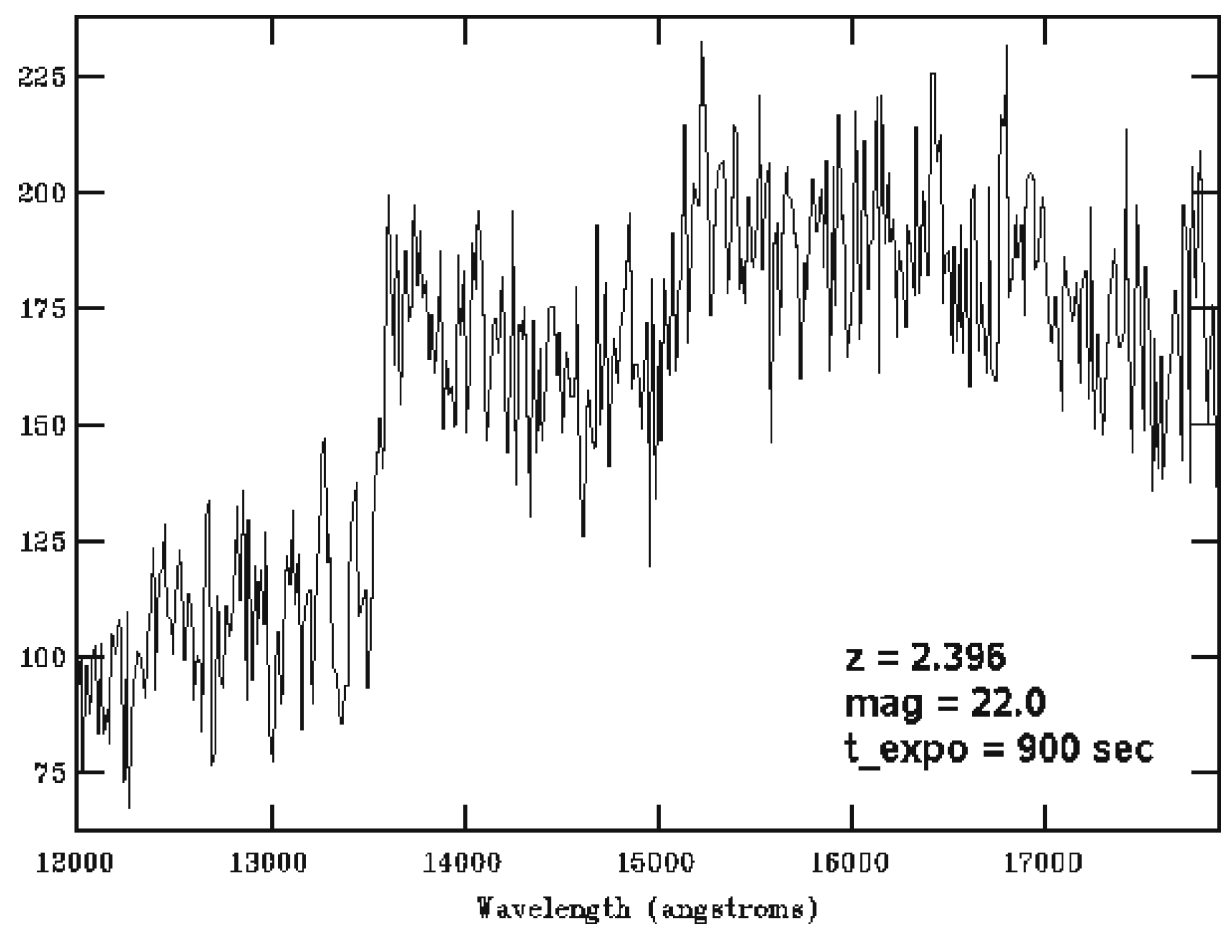

Fig. 5 Simulated spectrum of an early-type passively evolving galaxy at $z>2$. The D4000 break and the main absorption lines (CaII H\&K) are clearly detected. It is impossible to obtain such a spectrum with ground-based near-IR spectroscopy due to the sky background, $\mathrm{OH}$ line contamination and telluric absorptions

spectroscopic redshift measurements between $80 \%-99 \%$ for the planned integration times of $900 \mathrm{~s}$ (All-Sky Survey) and $7 \mathrm{~h}$ (Deep Survey) for all galaxy types and redshifts. The typical accuracy on individual redshifts $\sigma_{z}=0.001$. In comparison, with the same integration time of $900 \mathrm{~s}$, slitless spectroscopy would provide a $\mathrm{S} / \mathrm{N}$ ratio about 10 times lower.

\subsection{Imaging}

We verified that the photometric catalogues derived from SPACE imaging will provide high quality target lists for the spectroscopic observations. We used the NICMOS H-band observations of the Hubble Ultra Deep Fields (HUDF) with PSF $F W H M \sim 0.15^{\prime \prime}$ to match closely the PSF delivered by the $1.5 \mathrm{~m}$ SPACE mirror and resampled the data at the SPACE scale of $0.375^{\prime \prime} /$ pixel. The vast majority of sources detected in this image, reaching well beyond $H_{\mathrm{AB}}=27$, are still easily seen in the resampled image without blending or confusion. We measured blending and confusion as a function of magnitude using source detection software for the original and resampled HUDF images. The results are: (1) at the $H_{\mathrm{AB}}<23$ (all sky survey), $97 \%$ of the sources are still individually detected in the resampled image, (2) at $H_{\mathrm{AB}}=26$, only $\sim 15 \%$ of the sources appear blended with neighbouring sources, 
(3) source counts from the original and resampled image are consistent well beyond $H_{\mathrm{AB}}=26$.

\section{The $S P A C E$ survey programmes}

The main objective of SPACE will be to execute a Core Science Program in about 3.5 years. However, given the power and flexibility of SPACE in many fields of astrophysics, it will allow also for Guest Observer Science Programs. The Core Program will be composed of:

- The SPACE all-sky survey will observe the entire sky at galactic latitudes $> \pm 18^{\circ}$ ( $\sim 70 \%$ of the total). This corresponds approximately to 28,500 square degrees and 71,000 satellite pointing (1 pointing $\left.=0.4 \mathrm{deg}^{2}\right)$. Assuming $20 \mathrm{~min}$ per pointing and an observing efficiency of $75 \%$, this will require 2.7 years. The expected number of galaxy spectra with a $1 / 3$ target random sampling is $(50,000 / 3) \times 71,000 \times 0.4 \sim 0.5 \times 10^{9}$. The selection of the spectroscopic targets will be done taking an acquisition image in the $\mathrm{H}$-band at $A B \sim 23.5$ immediately before the spectroscopic observation. Targets will be selected using onboard software applied to the images taken with SPACE itself, following the approach currently adopted in ground-based spectroscopic surveys (e.g. VVDS, zCOSMOS). As a byproduct, the acquisition images will produce the deepest all-sky imaging survey at $|b|> \pm 18^{\circ}$.

- The SPACE Deep Survey will target a $10 \mathrm{deg}^{2}$ field (25 SPACE pointings) down to $H_{\mathrm{AB}}=25$, with a target sampling rate of $90 \%$, and without any color pre-selection. About 200,000 objects (stars+galaxies) are expected in $1 \mathrm{deg}^{2}$ at $H_{\mathrm{AB}}<25$. With a multiplex of $\sim 6000$ objects and an integration time of about $7 \mathrm{~h}$ per observation to reach a sufficient $\mathrm{S} / \mathrm{N}$, we need $\sim[(200,000 \times 0.4 \times$ $0.90) / 6000] \times 25$ pointings $\times 7 h \approx 5$ months. We recall that the $z>7$ galaxy candidates will be pre-selected using the SPACE imaging in $\mathrm{z}, \mathrm{J}, \mathrm{H}$ bands and will be compulsory targets repeated in each of the 12 spectroscopic observations done for each of the 25 pointings so that they will accumulate a total integration time of $(7 \mathrm{~h} \times 12)=84 \mathrm{~h}$ (per pointing) needed to reach a sufficient $\mathrm{S} / \mathrm{N}$ for $\mathrm{J}$, $\mathrm{H}=26$ (AB). The time needed for the broad-band z-, J- , H-band and narrowband imaging will be negligible with respect to the time dedicated to spectroscopy. With an appropriate strategy of repeated visits, the Deep Survey could be used also for detecting high-z SNe (see Section 5)

- The SPACE Galactic-plane survey will cover in Integral field mode the strip within $\pm 0.5^{\circ}$ centered on the Galactic Equator between $\pm 60^{\circ}$ of galactic longitude. We will allocate $4 \mathrm{hr}$ per pointing, i.e. $10 \mathrm{~h}$ per square degree and 50 days total for the entire survey.

Allocating a fraction of the time to a Guest Observer Program will add value to the mission. We will split the Core Science program in a four phases lasting approximately 9 months each, allowing the GO observing time to be ramped up over time in a manner similar to the phasing in past missions such as ISO and Spitzer. This will allow the core science program to be completed in a timely manner while providing the community with early instrument performance information and science results to use in the planning of observations for other studies. 


\section{SPACE mission concept}

\subsection{Driving requirements}

The science requirements identified in the previous sections place firm constraints on mission concept and the design of instrument.

The first need is to access the whole celestial sphere for about 3 years. The core observing program requires $0.1^{\prime \prime}$ pointing stability for at least $20 \mathrm{~min}$ of typical integration time, high temperature stability and good access to the ground station for data downlink and telemetry. All these requirements are ideally met by a largeamplitude halo orbit around the second Earth-sun Lagrange point (L2). A halo orbit at L2 maximizes the availability of the sky for observation with respect to the visual and thermal disturbance from the Sun, Earth and Moon. The thermal environment is very stable and, with the exception of solar storms, the radiation environment is relatively low. On a halo orbit of sufficient amplitude eclipses can be avoided altogether, while the constant satellite-sun-Earth geometry eases the thermal control and the communications. L2 is considered the ideal vantage point for an IR observatory and, following WMAP, several mission (e.g. Herschel, Planck, GAIA, JWST) will be operated at L2.

In order to measure a large number of sources, one needs multi-object spectroscopic capability over an area of the order of 1 square degree. In order to observe faint sources, one needs to operate in slit mode, exploiting the celestial background from space $\sim 500$ times lower than from the ground. The sensitivity requirement also drives the telescope diameter, whereas the large field of view drive the effective focal length of the optical system. The broad wavelength range and resolution of the spectrograph drives the choice of materials (mirrors vs. lenses), the detector technology, the instrument temperature, and ultimately the multiplexing capability of the instrument. In particular, the need for extensive multi-object capability suggests considering Micro-Electro-Mechanical-Systems (MEMS), following the approach pioneered for NIRSPEC on JWST [31, 35].

Having set through performance simulations the basic requirements on the instrument parameters (see Section 11), we have evaluated several possible solutions and converged finally on an elegant design that satisfies all requirements. To keep complexity and cost to a minimum we aimed for a single-mode instrument rather an all-purpose machine with a variety of arms and configurations. Nevertheless, we came out with a solution which inherently provides an extraordinary degree of versatility without adding complexity, weight or cost to the basic hardware needed for our main survey program. Using MEMS for target selection we have several millions of randomly addressable optical switches. Depending on the MEMS configuration, and on the selection of a filter or a prism in a filter wheel (we may have a single filter wheel, which would then represent the only moving part on SPACE) SPACE can perform imaging, slitless and slit spectroscopy (our main observing mode), and even wide-field integral field spectroscopy using Hadamard transforms.

\subsection{Payload description}

The SPACE payload is a compact and modular instrument, fitting below the primary mirror structure within a cylindrical envelope of $1.5 \mathrm{~m}$ diameter (the size of the 


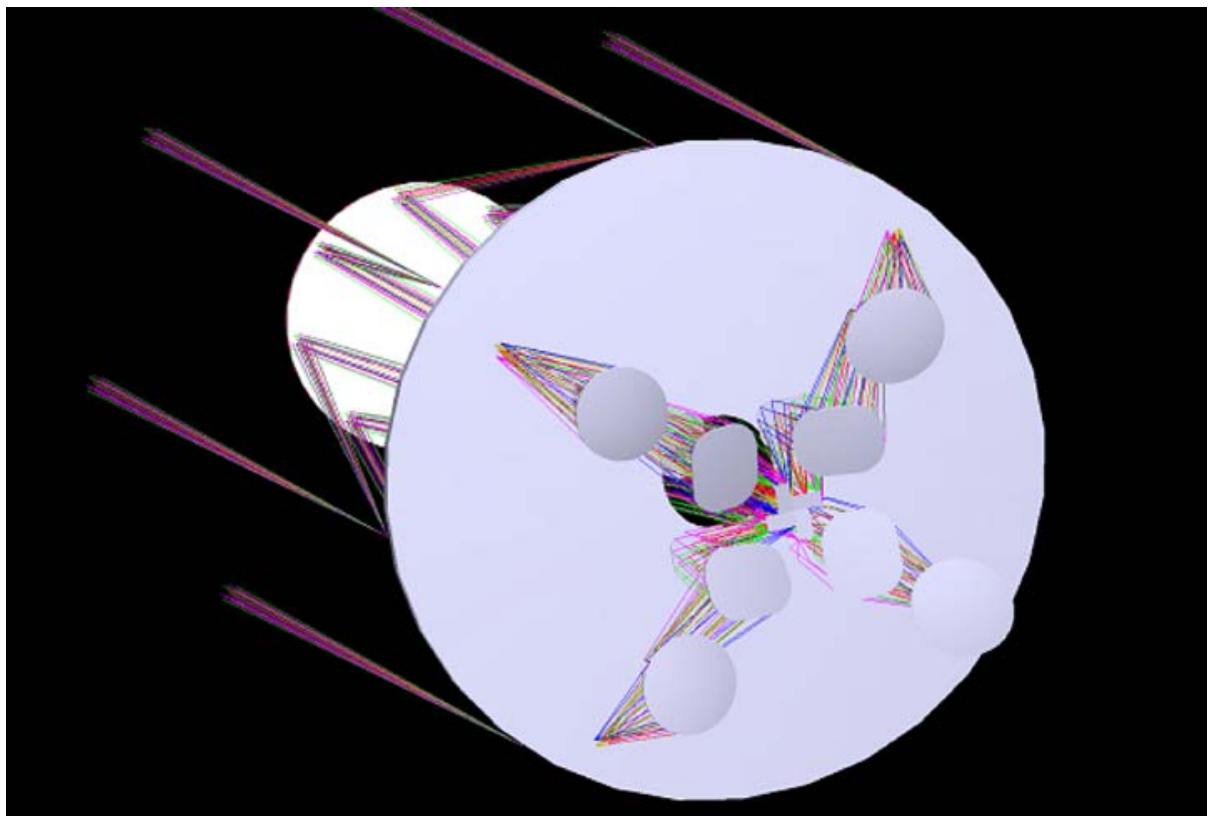

Fig. 6 Optical telescope assembly and fore-optics system (four channels)

primary) by $60 \mathrm{~cm}$ height. To accommodate for our large field of view, the beam is split into 4 identical channels, each one taking a $90^{\circ}$ sector of the cylinder. Each channel is a relatively conventional spectrograph with a MEMS device located at the intermediate focal plane. For the MEMS device we have opted for a Texas Instrument DMD of $2048 \times 1080$ micro-mirrors, whereas for the detector we use a $4 \mathrm{k} \times 4 \mathrm{k}$ infrared Focal Plan Assembly similar to the one developed for JWST. Each micro-mirror images a square $0.75^{\prime \prime} \times 0.75^{\prime \prime}$ field onto $2 \times 2$ detector pixels. Hereafter we will examine the baseline optical design and the main component of a spectrograph more in detail (Fig. 6).

1. The telescope is a Ritchey-Chrétien $\mathrm{F} / 5.83$, with a $1.5 \mathrm{~m} \mathrm{~F} / 2.7$ primary. A Ritchey-Chrétien telescope, in which both mirrors are hyperbolic, is free from coma and allows for easy optical testing.

2. The telescope focal plane falls on a 4-face pyramid mirror providing a total coverage of $51^{\prime} \times 27^{\prime}(\sim 0.4$ square degrees $)$. The layout sketched in Fig. 7 preserves the symmetry around the optical axis allowing the four channels to be identical with substantial cost saving in the design, fabrication and testing. It still allows to tile the sky with multiple contiguous observation to map large fields without significant losses in coverage.

3. Each subfields of the pyramid mirror is reflected toward a fore-optics system, made of 4 mirrors (Fig. 8) contained in a layer $\sim 400 \mathrm{~mm}$ deep under the primary support structure. The fore-optics system projects on the DMD an undistorted image of the focal plane with a scale of $0.75^{\prime \prime} / \mathrm{DMD}$ facet and excellent optical quality. 
Fig. 7 Four-fold symmetric cross for the SPACE pyramid mirror allowing for 4 identical optical channels

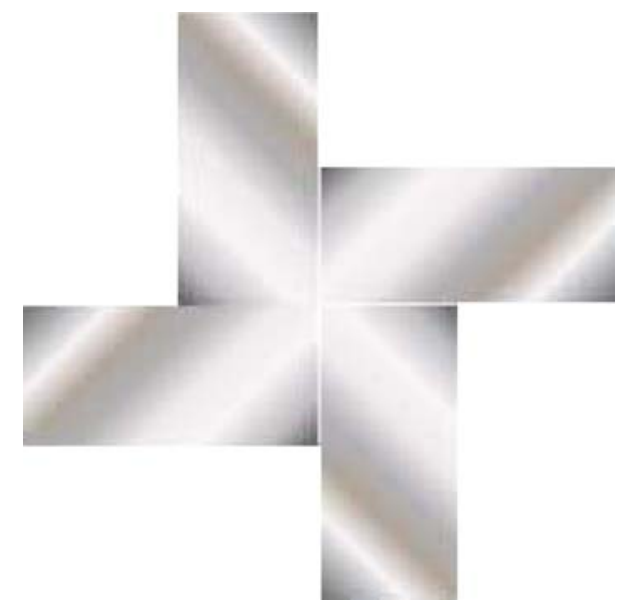

4. The DMD micro-mirrors are $13.6 \mu \mathrm{m}$ on a side and tilt along their diagonal by $\pm 12^{\circ}$. When the DMD facets are tilted to their ON position, the beam is reflected in a direction approximately normal to the DMD plane, while when they are turned OFF they reflect at an oblique angle towards a beam dump.

5. The collimator collects the beam reflected by the DMD relaying an image at infinity. Its design is similar to that of the fore-optics, with 4 mirrors located in the same $400 \mathrm{~mm}$ thick layer (Fig. 9). The collimator, however, develops inward along a cord rather than outward along a radius, therefore the incoming and outcoming beams do not cross. The collimator produces a $66 \mathrm{~mm}$ pupil located nearly on the same plane of the pyramid mirror.

6. The prism is located in the immediate vicinity of the pupil. Having set at $R \sim 400$ target, we have considered a number of solutions based on both grisms and prisms in different configurations and materials. Our baseline choice is for a single pass prism + folding mirror placed immediately after the pupil. For the imaging mode, the prism + folding mirror are substituted by a simple $45^{\circ}$ folding mirror and broad-band filter. A single filter wheel per channel allows to insert the other required imaging filters (e.g. z, J, narrow band).

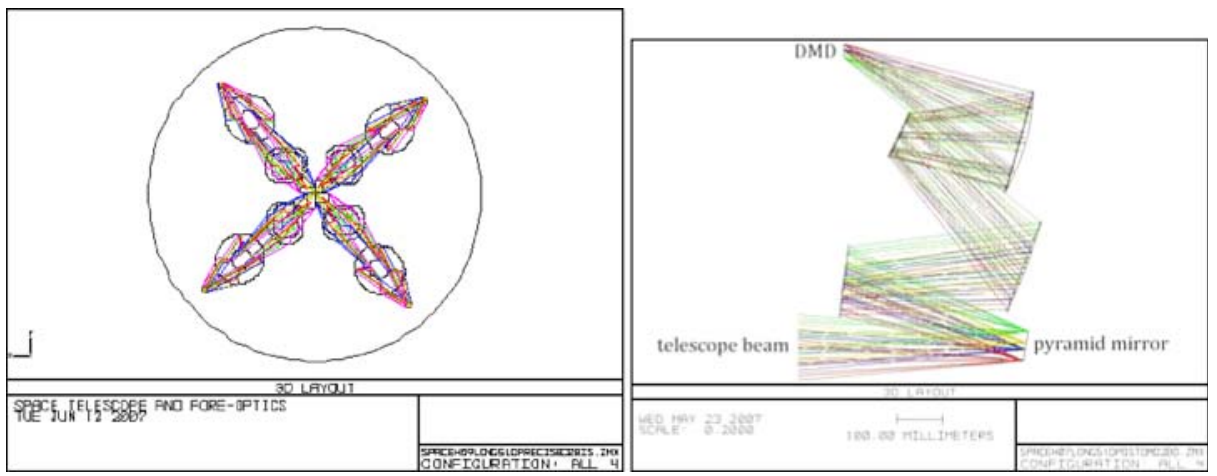

Fig. 8 Fore-optics system. Left: top view (all channels); Right: side view (one channel) 

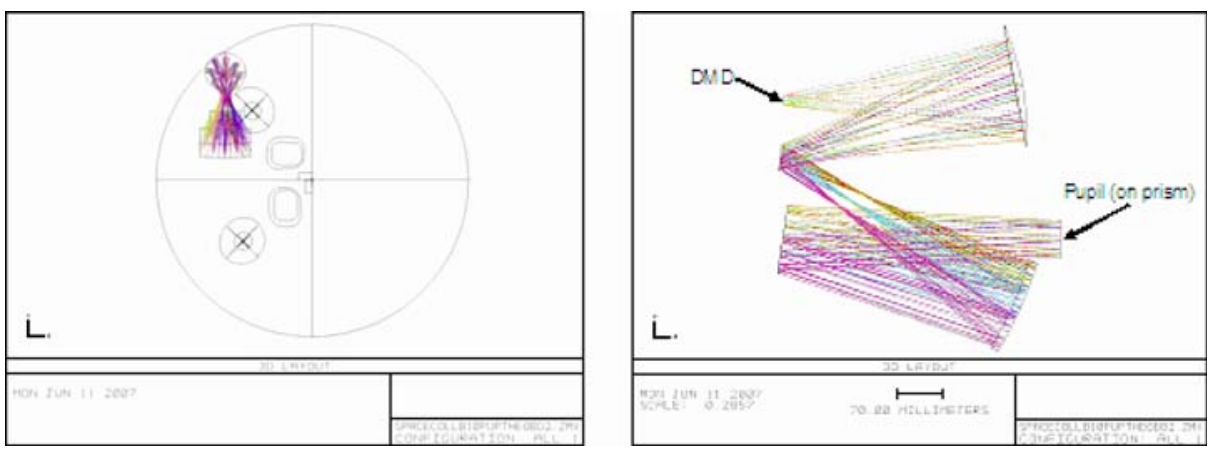

Fig. 9 Left: top view layout of the collimator (one channel). The location of the fore-optics mirrors of two channels (Fig. 8) is also shown to illustrate the different orientation of the collimator (along a cord) with respect to the fore-optics (along a radius) after reflection on the DMD. Right: side view layout of the collimator

7. The camera reimages the DMD plane onto the detector plane with a 1:2 ratio. The camera has 4 lenses, including 2 aspheric surfaces, and is achromatic and diffraction limited between 0.6 and $1.8 \mu \mathrm{m}$. The length of a $0.8-1.8 \mu \mathrm{m} R \sim 400$ spectrum is $\sim 670$ pixels, slightly dependent on the field position. Thanks to a folding mirror, the detector can be located in the area below the pyramid mirror. Having all IR detectors located close to each other simplifies the cooling system (Fig. 10).

Fig. 10 Prism and camera 3-d layout. A $45^{\circ}$ folding mirror can be inserted in the camera to relay the image in the most convenient position at center of the spacecraft

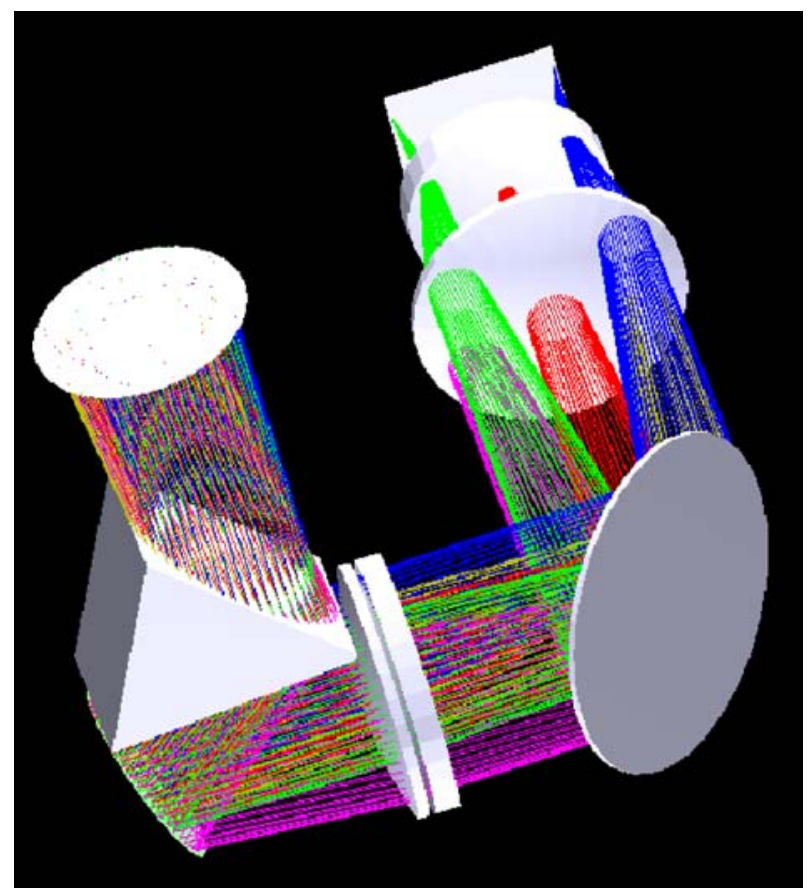


Fig. 11 A $2 \mathrm{k} \times 2 \mathrm{k}$ buttable Focal Plan Array developed for JWST

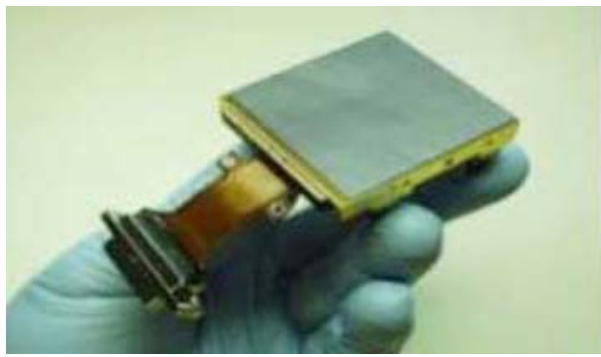

\subsection{Detectors}

SPACE will utilize $\mathrm{HgCdTe}$ Focal Plane Arrays (FPAs). Teledyne devices, based on the Hawaii-2RG multiplexer, with long wavelength cut-off at $\sim 1.8 \mu \mathrm{m}$ are ideally suited because of their modest sensitivity to the spacecraft thermal emission and to the low dark current levels exhibited at relatively high operating temperatures, $\sim 140-150 \mathrm{~K}$, but more conventional $2.5 \mu \mathrm{m}$ devices can also be used, since the spacecraft will be passively cooled. This technology is mature. The IR channel of the HST/WFC3 camera is equipped with FPAs with $1.72 \mu \mathrm{m}$ cutoff wavelength on a $1 \mathrm{k} \times 1 \mathrm{k}$ multiplexer. The most recent WFC3 parts attain $\sim 80 \%$ QE nearly flat over a $1.0-1.70 \mu \mathrm{m}$ range, readout noise in double correlated sampling $<25$ e/read and dark current $\sim 0.01 \mathrm{e} / \mathrm{s} / \mathrm{pixel}$ at $140 \mathrm{~K}$. JWST devices for NIRCAM and NIRSPEC with $2.5 \mu \mathrm{m}$ cutoff have also been produced in $2 \mathrm{k} \times 2 \mathrm{k}$ format, with excellent performance. Note that the substrate removal process, necessary to eliminate radiation induced glow, extends the spectral response down to $0.4 \mu \mathrm{m}$, allowing an extension of the wavelength range down to e.g. $0.60 \mu \mathrm{m}$ that we are considering for SPACE (Fig. 11).

These devices can be butted together in a $2 \times n$ configuration (Focal Plane Assembly, FPA). For SPACE we are considering $2 \times 2$ FPA similar to those developed for JWST, A $4 \mathrm{k} \times 4 \mathrm{k}$ assembly allows to easily accomodate for the extension of the spectra.

\subsection{Digital micromirror devices (DMDs)}

The innovative technical aspect of the SPACE mission is the use of DMDs. We have taken a fresh look at these devices, that were originally considered for NIRSPEC on JWST, and concluded that they represent the ideal choice for SPACE. Since their invention by L. Hornbeck at Texas Instruments (TI) in 1988, DMD have been serially produced with volumes exceeding 10 million units shipped by 2006. Today DMDs represent one of the leading technologies in digital imaging (e.g. DLP projectors). For this multi-billion dollar market huge investments have been made, reaching unique levels of quality and reliability. We intend to capitalize on these investments (Fig. 12). 

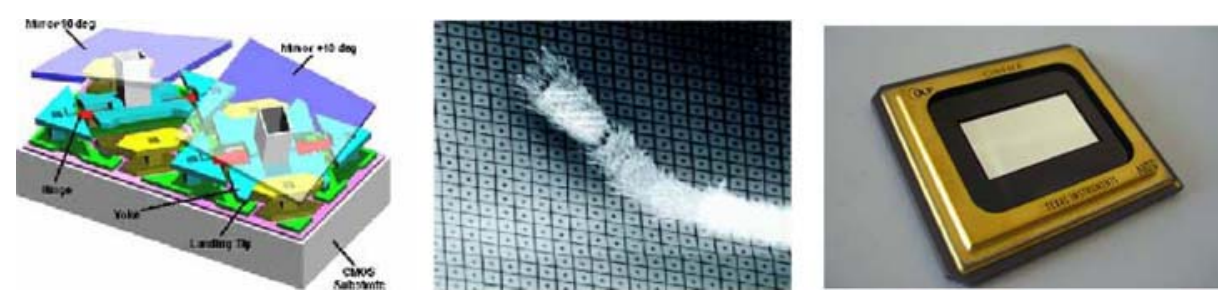

Fig. 12 Left: typical substructure of a TI DMD; center: DMD array with an ant leg for comparison; 3: packaged DMD CINEMA $(2048 \times 1080)$ device

\subsubsection{What are DMDs?}

DMDs are a particular type of MEMS. MEMS integrate microscopic mechanical elements, in our case moving mirrors, together with their control electronics on a common silicon substrate. Whereas the control electronics is fabricated using standard integrated circuit processes, the mechanical components are fabricated using "micromachining" processes that selectively etch away parts of the silicon wafer or add new structural layers. In the case of DMDs, the mechanical part is an array of up to 2.21 million aluminum micromirrors fabricated on top of a complementary metal oxide semiconductor static random access memory array. Each micromirror, independently controlled, can switch along its diagonal thousands of times per second as a result of electrostatic attraction between the mirror structure and the underlying electrodes. The current generations of DMDs all exhibit a $+12^{\circ} /-12^{\circ}$ tilt angle, limited by a mechanical stop. Pixel pitch in currently available DMD is either $10.8 \mu \mathrm{m}(0.6 \mu \mathrm{m}$ gap width $)$ or $13.68 \mu \mathrm{m}(0.68 \mu \mathrm{m}$ gap width $)$ center-to-center. TI produces several types of DMDs in hermetic packages. Those with more than 2 million pixels are the $2048 \times 1080$ pixels CINEMA device (Fig. 12) with $13.68 \mu \mathrm{m}$ pixel pitch, and the $1920 \times 10801080$ p High Definition TV device with $10.6 \mu \mathrm{m}$ pixel pitch. For our baseline design we have considered the former, but optical solutions can be found for smaller devices.

\subsubsection{Why DMDs? (instead of micro-shutter arrays)}

The main alternative to DMD is the Micro-shutter array (MSA), which has been developed and optimally matched to the IRMOS/JWST. MSAs represent an outstanding technological achievement and are especially unique in their capability of operating at low temperatures. However, they come in much smaller size (at the moment $171 \times 365$ pixels) and are still prone to cosmetic defects. Other figures of merit (e.g. filling factor, contrast, reliability) do not appear to be superior to those achieved by the current generation of commercial DMDs. DMDs are technologically mature, already in use in Astronomy [32] and meet all the optical and technical needs of the SPACE mission. The issues (optical quality, constrast, reliability, radiation environment, ....) related to the use of DMD in our mission are discussed in detail in the SPACE proposal. 


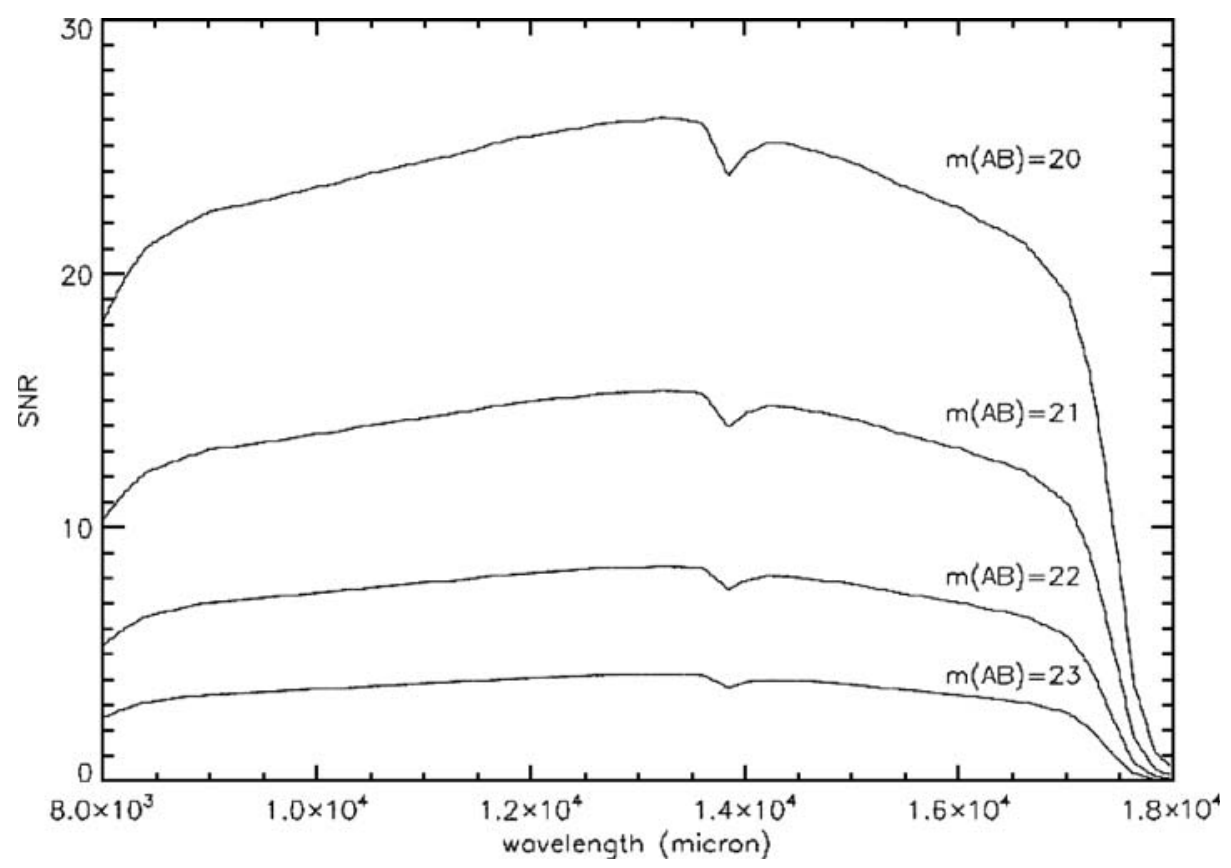

Fig. 13 Estimated SNR of SPACE spectra in $900 \mathrm{~s}$ integration. We used detector parameters typical of WFC3/IR flight candidates. Transmission efficiencies of all reflective and refractive components are those of the WFC3/IR optical coating. Light losses due to the prism thickness are included, together with the most recent zodiacal background prediction for the SNAP mission also at L2

\section{Performance assessment with respect to science objectives}

We have verified that with our proposed design SPACE reaches the sensitivity goals stated in the Sections 11 and 12. Using the baseline optical design described in the previous section we have estimated the signal to noise ratio per resolution element in $900 \mathrm{~s}$ of integration (Fig. 13). A $S N R \sim 3$ at $\mathrm{AB}=23$ is the sensitivity requirement derived in Section 3 for the All-sky survey. The sensitivity estimates for the Deep Survey are also compatible with the requirements.

\section{Operating modes}

The all-sky survey will be performed repeating the same basic observing mode, sketched in Fig. 14. After locking the guider, a $\sim 30 \mathrm{~s}$ broad-band image will be taken and corrected for dark, bad pixels and flat field using calibration frames stored on-board. A software routine (similar to the one we used in Section 11.1) will then automatically select the targets, generate and upload the optimal DMD configuration. In the mean time, the prism will be inserted at the place of the broadband filter. We allocate $5 \mathrm{~min}$ for these operations, which will proceed in parallel on the four channels. 

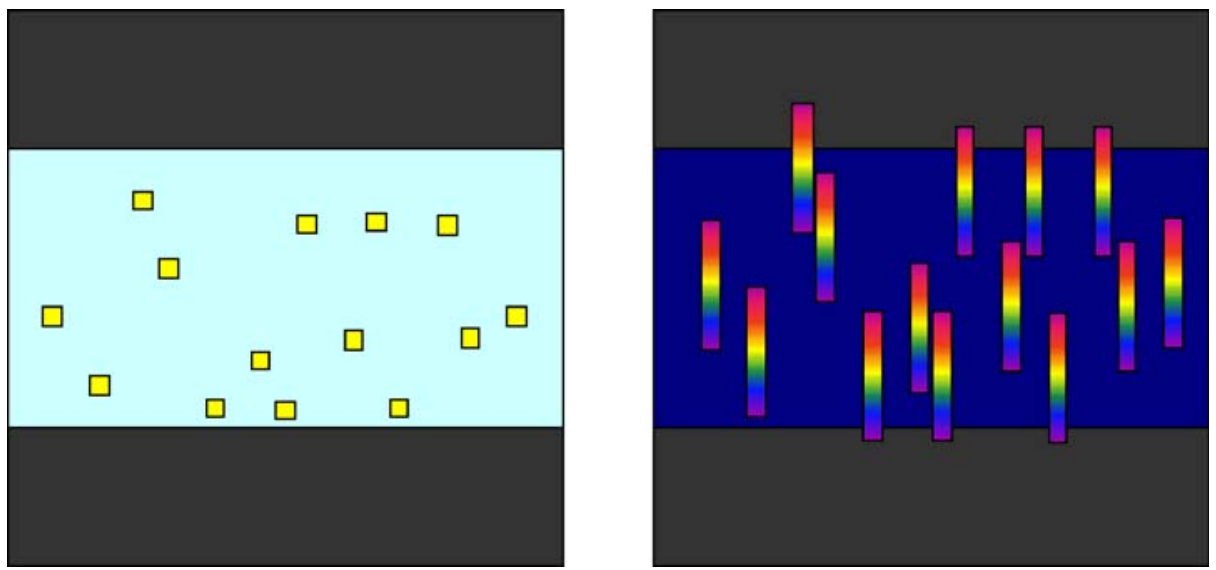

Fig. 14 Sketch of the data acquisition and observing procedure. Left: the DMD field is projected onto the detector (dark background) in broad band imaging mode. The light blue color indicates the high background, targets are represented by yellow squares. Right: all DMDs are turned off except those of the targets, the prism is inserted. The background is low (dark blue) and spectra are produced

As mentioned in Section 11, refinements are possible, e.g. splitting the acquisition of spectra in multiple exposures to eliminate cosmic rays or to optimize the extraction of the bright sources. The HAWAII 2RG detectors allow to address and read individual pixels while the others integrate (guide mode). This capability may also be exploited to synchronize the readout of certain areas with the opening of the corresponding slits.

The long exposures performed for the Deep Survey will be more efficient, avoiding the frequent broad-band acquisition. We plan to add telescope dithering to recover spatial resolution and dead pixels. For the integral field spectroscopic survey of the Galactic plane, we will use Hadamard transform spectroscopy. Proposed by J. Mather for NIRSPEC on JWST and successfully validated with IRMOS (Fixsen and MacKenty, manuscript in preparation), this technique can be regarded as a variation of long slit spectroscopy. By opening multiple slits at the same time, overlapped spectra are obtained. By using different slit patterns, the entire set of overlapped spectra can be eventually disentangled and the entire data cube $(x, y, \lambda)$ reconstructed. Our estimates for the Galactic plane survey allocates $4 \mathrm{~h}$ per pointing, which corresponds to individual exposures of the order of order of $\sim 15 \mathrm{~s}$. The lower signal to noise of the raw images will allow for a more robust compression factor than the noiseless $5 \times$ used for the standard observations. The actual implementation of the Hadamard transform mode will require further analysis.

\section{Mission operations and data analysis}

Soyuz-Fregat is our proposed launcher. Soyuz, equipped with the Fregat upper stage, launched from Kourou, is capable of delivering a 2-ton spacecraft directly into L2 transfer orbit without any need for large on-board propulsion. A typical duration of the transfer is 4 months. Once L2 has been reached, the delta-v requirements for 
orbit maintenance and formation keeping are small, a few $\mathrm{m} / \mathrm{s} / \mathrm{year}$. The launcher provides a fairing $3.8 \mathrm{~m}$ wide and up to $8.5 \mathrm{~m}$ high (with $14.5^{\circ}$ taper above $5 \mathrm{~m}$ ), which can easily accommodate the spacecraft configuration with an external baffle for stray light rejection.

In the survey phase, the sequence of operations is particularly simple (20 to 30 pointing, small slew, new pointing, and so on), easily automated and managed. Targeted observations will require similar sequences. A ground station such as Cebreros or New Norcia will have L2 above the horizon for more than $8 \mathrm{~h}$ per day on average, with a minimum of $6 \mathrm{~h}$ /day in the most unfavorable season for the given location in the Northern or Southern hemisphere. Our most conservative assumptions on compression (5 Mbit/s) and transmission (1.5 Mbit/s) require a station scheduled $3.5 \mathrm{~h}$ per day for SPACE.

SPACE telemetry and command will be received/transmitted by a dedicated antenna at a single ESA Ground Station having the task of gathering the downlinked housekeeping and science telemetry and uploading the commands needed to control spacecraft and payload. The Ground Station will be shared with other missions having similar visibility requirements. The telemetry will be sent through a dedicated redundant line of appropriate bandwidth to a Mission Operations Centre (MOC) under the control of ESA. The MOC is in charge of checking the housekeeping telemetry to verify the safety of spacecraft and payload, taking if needed the appropriate corrective actions by means of commands sent following pre-specified procedures. The stream of housekeeping and science telemetry, through an ESAprovided dedicated redundant line of appropriate bandwidth, will be then ingested into a Science Operations Centre (SOC) under the control of the SPACE Consortium. The SOC will also generate the commands needed for the satellite pointing and observations, and send them to the MOC in the form of agreed-upon procedures.

The SOC will distribute the data to a Science Data Centre (SDC), which will focus on the spectra contained in the images, their extraction, analysis, distribution, archiving and generation of scientific results. The SDC will represent the interfaces between the project and the scientific community. The SDC will receives both the raw data and pre-reduced data from the SOC, adding all services needed to efficiently exploit their science content.

Acknowledgements The authors acknowledge the support from the University of Bologna and the Space Telescope Science Institute. Thales Alenia - Milano (Italy) is warmly acknowledged for its contributions and support.

\section{References}

1. Albrecht, A., et al.: Dark Energy Task Force Report. http://home.fnal.gov/ rocky/DETF/ (2006)

2. Aldering, G.: SNAP Sky Background at the North Ecliptic Pole. LBNL report number LBNL$51157(2001)$

3. Amendola, L.: Coupled quintessence. Phys. Rev. D 62, 3511 (2000)

4. Angulo, R., et al.: Constraints on the dark energy equation of state from the imprint of baryons on the power spectrum of clusters. MNRAS 362, L25 (2005)

5. Angulo, R., et al.: The detectability of baryonic acoustic oscillations in future galaxy surveys. MNRAS 338, 755 (2008)

6. Astier, P., et al.: The supernova legacy survey: measurement of $\Omega_{M}, \Omega_{\Lambda}$ and $\mathrm{w}$ from the first year data set. A\&A 447, 31 (2006) 
7. Baugh, C.M.: A primer on hierarchical galaxy formation: the semi-analytical approach. Rep. Prog. Phys. 69, 3101 (2006)

8. Blake, C., Glazebrook, K.: Probing dark energy using baryonic oscillations in the galaxy power spectrum as a cosmological ruler. ApJ 594, 665 (2003)

9. Bottini, D., et al.: The very large telescope visible multi-object spectrograph mask preparation software. PASP 117, 996 (2005)

10. Bouwens, R.J., Illingworth, G.D., Blakeslee, J.P., Franx, M.: Galaxies at z $\sim 6$ : the UV luminosity function and luminosity density from 506 HUDF, HUDF parallel ACS field, and GOODS i-Dropouts. ApJ 653, 53 (2006)

11. Bouwens, R., et al.: Galaxies at z 7-8: z850-dropouts in the hubble ultra deep field. ApJ 616, L79 (2004)

12. Bouwens, R.J., Illingworth, G.D., Thompson, R.I., Franx, M.: Constrainsts on $\mathrm{z} \sim 10$ galaxies from the deepest hubble space telescope NICMOS fields. ApJ 624, L5 (2004)

13. Capozziello, S., Francaviglia, M.: Extended theories of gravity and their cosmological and astrophysical applications. GReGr 40, 357 (2008)

14. Choudhury, T.R., Ferrara, A.: Updating reionization scenarios after recent data. MNRAS 371, L55 (2006)

15. Eisenstein, D., et al.: Detection of the baryon acoustic peak in the large-scale correlation function of SDSS luminous red galaxies. ApJ 633, 560 (2005)

16. Eisenstein, D., et al.: Improving cosmological distance measurements by reconstruction of the baryon acoustic peak. ApJ 664, 675 (2006)

17. Fan, X., et al.: A survey of $z>5.7$ quasars in the sloan digital sky survey. III. Discovery of five additional quasars. AJ 128, 515 (2004)

18. Guzzo, L., et al.: A test of the nature of cosmic acceleration using galaxy redshift distortions. Nature 451, 541 (2008)

19. Hamilton, A.J.S.: Precision measurement of large scale structure. Technical report, NASA GSFC (2001)

20. Hu, W., Haiman, Z.: Redshifting rings of power. PhRvD 68, 6 (2003)

21. Huff, E., et al.: Simulations of baryon oscillations. APh 26, 351 (2007)

22. Hütsi, G.: Acoustic oscillations in the SDSS DR4 luminous red galaxy sample power spectrum. A\&A 449, 891 (2005)

23. Hütsi, G.: Power spectrum of the SDSS luminous red galaxies: constraints on cosmological parameters. A\&A 459, 375 (2006)

24. Kashlinsky, A.: Cosmic infrared background and early galaxy evolution. PhR 409, 361 (2005)

25. Ketchum, W., Wang, Y: Submitted to ApJ (2007)

26. Kinney, A., Calzetti, D., Bohlin, R.C., et al.: Template ultraviolet to near-infrared spectra of star-forming galaxies and their application to k-corrections. ApJ 467, 38 (1996)

27. Linder, E.: Cosmic growth history and expansion history. Phys. Rev. D 72(4), id. 043529 (2005)

28. Linder, E.: The mirage of $\mathrm{w}=-1$. arXiv:0708.0024

29. Linder, E.: Redshift distortions as a probe of gravity. arXiv:0709.1113

30. Lue, A., Scoccimarro, R., Starkman, G.D.: Probing Newton's constant on vast scales: DvaliGabadadze-Porrati gravity, cosmic acceleration, and large scale structure. Phys. Rev. D 69, 124 015 (2004)

31. MacKenty, J.W., Stiavelli, M.: A multi-object spectrometer using micro mirror arrays. ASP Conf. 195, $443(2000)$

32. MacKenty, et al.: Commissioning of the IRMOS MEMS spectrometer. SPIE 6269, 37M (2006)

33. Mannucci, F., Basile F., Poggianti, B.: Near-infrared template spectra of normal galaxies: k-corrections, galaxy models and stellar populations. MNRAS 326, 745 (2001)

34. Martini, P., DePoy, D.L.: Optimal resolutions for IR spectroscopy through the OH airglow. Proc. SPIE 4008, 695 (2000)

35. Moseley, S.H., et al.: High-efficiency, wide-band, multi-object, near-infrared spectrograph for the NGST. ASP Conf. 207, 262 (2000)

36. Mullis, C., et al.: Discovery of an X-ray- luminous galaxy cluster at $\mathrm{z}=1.4$. ApJ 623, L85 (2005)

37. Padmanabhan, T.: Dark energy and gravity. arXiv0705.2533 (2007)

38. Peacock, J.A., et al.: ESA-ESO working group report on fundamental cosmology, astro$\mathrm{ph} / 0610906$ (2006)

39. Percival, W.J., et al.: Measuring the baryon acoustic oscillation scale using the sloan digital sky survey and 2df galaxy redshift survey. MNRAS 381, 1053 (2008)

40. Perlmutter, S., et al.: Measurements of omega and lambda from 42 high-redshift supernovae. ApJ 517, 565 (1999) 
41. Riess, A.G., et al.: Observational Evidence from supernovae for an accelerating universe and a cosmological constant. AJ 116, 1009 (1998)

42. Riess, A.G., et al.: New hubble space tele scope discoveries of type Ia supernovae at $\mathrm{z}>=1$ : narrowing constraints on the early behavior of dark energy. ApJ 659, 98 (2007)

43. Rosati, P. et al.: Chandra and XMM-Newton Observations of RDCS 1252.9-2927, A massive cluster at $\mathrm{z}=1.24$. AJ 127, 230 (2004)

44. Salvaterra, R., Magliocchetti, M., Ferrara, A., Schneider, R.: The infrared glow of the first stars. MNRAS 368, L6 (2006)

45. Sanchez, A.G., et al.: Cosmological parameters from cosmic microwave background measurements and the final $2 \mathrm{dF}$ galaxy redshift survey power spectrum. MNRAS, 366, 189 (2006)

46. Scannapieco, E., Schneider, R., Ferrara A.: The detectability of the first stars and their cluster enrichment signatures. ApJ 589, 35 (2003)

47. Schaerer, D.: The transition from Population III to normal galaxies: Lyalpha and He II emission and the ionising properties of high redshift starburst galaxies. A\&A 397, 527 (2003)

48. Shapley, A., Steidel, C.C., Pettini, M. et al.: Rest-frame ultraviolet spectra of z 3 Lyman break galaxies. ApJ 588, 65 (2003)

49. Seo, H.-J., Eisenstein, D.: Probing dark energy with baryonic acoustic oscillations from future large galaxy redshift surveys. ApJ 598, 720 (2003)

50. Seo, H.-J., Eisenstein, D.: Probing dark energy with baryonic acoustic oscillations. ApJ 633, 575 (2005)

51. Sigad, Y., et al.: Measuring the nonlinear biasing function from a galaxy redshift survey. ApJ 540, $62(2000)$

52. Simcoe, R.A.: High-redshift intergalactic C IV abundance measurements from the near-infrared spectra of two z 6 QSOs. AJ 653, 977 (2006)

53. Spergel, D.N., et al.: Three-year wilkinson microwave anisotropy probe (WMAP) observations: implications for cosmology. ApJS 170, 377 (2007)

54. Stanford, S.A., et al.: The XMM cluster survey: a massive galaxy cluster at $\mathrm{z}=1.45$. ApJ 646, L13 (2006)

55. Tegmark, M.: Measuring spacetime: from the big bang to black holes. Science 296, 1427 (2003)

56. Tegmark, et al.: Cosmological constraints from the SDSS luminous red galaxies. PhRvD 74, 12 (2006)

57. Thompson, R., et al.: Evidence for a $\mathrm{z}<8$ origin of the source-subtracted near-infrared background. ApJ 657, 669 (2007)

58. Trotta, R.: Probing dark energy with future surveys. arXiv:astro-ph/0607496 (2006)

59. Venemans, B., et al.: The discovery of the first luminous $\mathrm{z} \sim 6$ quasar in the UKIDSS large area survey. MNRAS, 376L, 76 (2007)

60. Verde, L., et al.: The $2 \mathrm{dF}$ galaxy redshift survey: the bias of galaxies and the density of the universe. MNRAS 335, 432 (2002)

61. Vestergaard, M.: Determining central black hole masses in distant active galaxies. ApJ 571, 733 (2002)

62. Wang, Y.: Dark energy constraints from baryon acoustic oscillations. ApJ 647, 1 (2006)

63. Wang, Y.: Differentiating dark energy and modified gravity with galaxy redshift surveys. arXiv:0710.3885 (2007)

64. Wang, Y., Mukherjee, P.: Observational constraints on dark energy and cosmic curvature. PRD 76, 10 (2007)

65. White, S.D.M., Rees, M.: Core condensation in heavy halos - a two-stage theory for galaxy formation and clustering. MNRAS 183, 341 (1978)

66. Willott, et al.: First results from the Canada-France high-z quasar survey: constraints on the $\mathrm{z}=$ 6 quasar luminosity function and the quasar contribution to reionization. ApJ 633, 630 (2005)

67. Wood-Vasey, W.M., et al.: Observational constraints on the nature of dark energy: first cosmological results from the essence supernova survey. ApJ 666, 694 (2007) 